\title{
Successful treatment of relapsed testicular embryonal rhabdomyosarcoma with Endostar and traditional chemotherapy: a case report
}

This article was published in the following Dove Press journal:

OncoTargets and Therapy

\author{
Tao Han ${ }^{1, *}$ \\ Jianjun Chen ${ }^{1, *}$ \\ Yuting Luan ${ }^{2, *}$ \\ Xiaoxia Chen ${ }^{1, *}$ \\ Xiaodan Yang' \\ Yue Zhang' \\ $\mathrm{Gao} \mathrm{Li}^{2}$ \\ Di Wang ${ }^{3}$ \\ Zhendong Zheng' \\ 'Department of Oncology, Cancer \\ Center of People's Liberation Army, \\ General Hospital of Shenyang Military \\ Region, Shenyang I I 0840, People's \\ Republic of China; ${ }^{2}$ Department \\ of Clinical Pharmacy, Shenyang \\ Pharmaceutical University, Shenyang \\ I I 0840, People's Republic of China; \\ ${ }^{3}$ Department of Pathology, General \\ Hospital of Shenyang Military Region, \\ Shenyang I I0840, People's Republic \\ of China
}

*These authors contributed equally to this work
Correspondence: Zhendong Zheng Department of Oncology, PLA Cancer Center, General Hospital of Shenyang Military Region, No 83, Wenhua Road, Shenhe District, Shenyang City, Liaoning Province I I0840, People's Republic of China

Email zhengzhdong@163.com

\begin{abstract}
Embryonal rhabdomyosarcoma (ERMS) has a low prevalence, poor prognosis, and limited treatment efficacy. We report a case of an 18-year-old male whose disease relapsed in the abdominal cavity after a testicular ERMS curative resection. The patient received eight sequential cycles of rescue therapy using cisplatin and isocyclophosphamide in combination with a vascular targeted drug, Endostar. The therapeutic effect of the combination regimen has been evaluated for complete response. This is the first case to report using Endostar and chemotherapy in relapsed ERMS, and the curative effect results in complete response. Endostar, a new vascular targeted drug, combined with chemotherapy may play a synergistic role and provide a reference for the treatment of ERMS.
\end{abstract}

Keywords: embryonal rhabdomyosarcoma, metastasis, complete response, endogenous angiogenesis inhibitor

\section{Introduction}

Embryonal rhabdomyosarcoma (ERMS), a rare category of soft tissue sarcoma (STS), originates in the mesenchymal tissue. There are three major histologic subtypes of rhabdomyosarcoma (RMS), embryonal, alveolar, and pleomorphic, and the first two are also called nonpleomorphic RMS. ${ }^{1,2}$ The head and neck region is the most affected site, followed closely by the genitourinary tract, in which $\sim 25 \%$ of cases occur. ${ }^{3}$ Surgery is the best method for removing early-stage tumors; however, there remains a high risk of recurrence or distant metastasis afterward. Despite clinical trials demonstrating the efficacy of many types of chemotherapy, ERMS has a low objective response rate to cytotoxic drugs and a poor long-term prognosis with short progression-free survival (PFS). ${ }^{4}$

Endostar was first approved by the China Food and Drug Administration in 2005 for the treatment of non-small-cell lung cancer. It is a broad-spectrum endogenous angiogenesis inhibitor and primarily acts on $\mathrm{a} 5 \mathrm{~b} 1 \mathrm{and}$ avb3 integrins, surface nucleolin, caveolin-1, glypicans-1 and 4, MMP-2, and the VEGFR-2 spectrum. ${ }^{5}$ Endostar selectively acts on microvascular endothelial cells and plays antiproliferation, antimigration, and apoptosis-promoting roles. ${ }^{6}$ In addition, a multicenter Phase II clinical trial was launched on April 20, 2017 to compare the efficacy and safety of Endostar combined with chemotherapy vs chemotherapy alone in patients with stage IV STSs (NCT03121833). Thus, Endostar combined with chemotherapy may theoretically improve patient prognosis.

Here, we report a case of an 18-year-old male with ERMS distant metastasis after surgery who was treated with chemotherapy combined with Endostar, and the patient showed complete response (CR) after eight sequential cycles. 


\section{Case report}

An 18-year-old Chinese man arrived at our hospital with fever, abdominal distension, abdominal pain, anorexia, and weight loss of $10 \mathrm{~kg}$ over the last 6 months. Upon physical examination, the patient had a Karnofsky performance score of 70 .

Six months previously, the patient had undergone testicular orchiectomy. We learned about the procedure from the surgical records. The doctor opened the right scrotum until the right testicle was revealed, and there were multiple solid tumors that presented sarcomatoid changes. The tumors had invaded the epididymis and surrounding spermatic cord upward into the penis and were considered malignant. After obtaining consent from the patient and his family, a radical resection of the tumor was performed. A portion of the tumor was examined, the size of which was $1.5 \times 1.0 \times 0.7 \mathrm{~cm}$, and the tumor was mostly covered with capsules. Under the microscope, concentrated and sparse areas of tumor cells alternated, with an oval shape or short spindles, and the cytoplasm was not obvious. Immunohistochemistry results demonstrated CK(-), Vimentin(+), PLAP(-), CD30(-), SALL4(-), MyoD1(+), Myogenin(+), Desmin(+), CD3(-), CD20(-), Pax-5 and Ki-67(+>50\%). We did not find evidence of retroperitoneal lymphadenopathy. The histopathological examination of the specimen revealed it to be ERMS $\mathrm{T}_{1} \mathrm{~N}_{\mathrm{x}} \mathrm{M}_{0}$ (Figure 1). Positron emission tomography (PET)/computed tomography (CT) showed multiple brown fat tissues all over the body and no abnormal metabolism at the operative site, and no tumor tissue was observed after surgery. Unfortunately, he and his family refused adjuvant therapy.

During this hospitalization, we discovered that the abdomen and pelvis had neoplasms, with a diameter

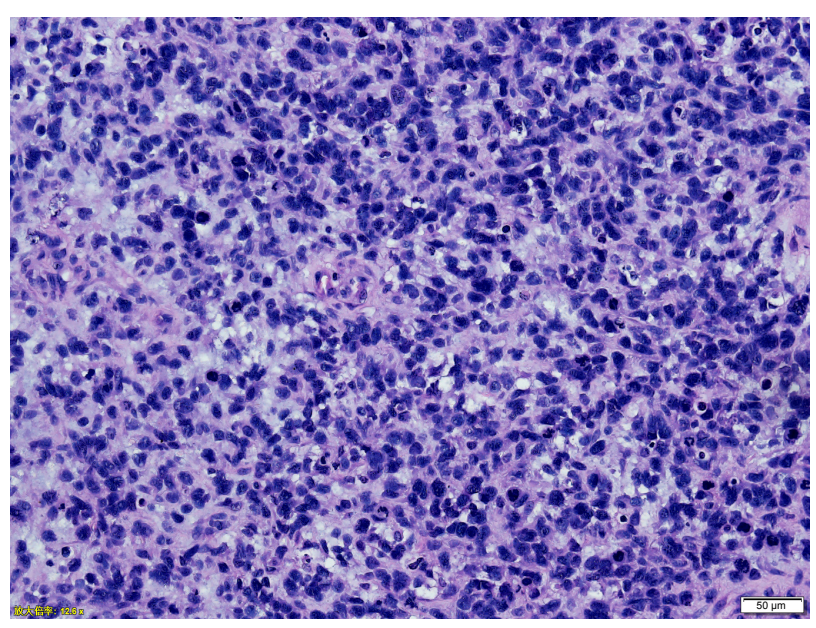

Figure I Histological section with haematoxylin and eosin staining, magnification, $\times 200$. Primarily round and spindle cells, were identified to contain eccentric nuclei and deeply eosinophilic cytoplasm. of $\sim 31.6 \times 10 \mathrm{~cm}$ by CT (Figure $2 \mathrm{~A}$ ). The image shows an abdominal tumor attached to the intestine. There were no other sites of metastasis.

Then, the patient was treated with a cross-line rescue therapy combining cisplatin and isocyclophosphamide and simultaneously with Endostar for a total of eight cycles. The method of administration is described in Table 1. During treatment, the patient experienced chemotherapy-related adverse reactions such as marrow suppression and nausea after two cycles, but the reactions were tolerable without adjusted doses. After the second and fourth cycles, the CT review showed that the abdominal and pelvis neoplasms were significantly reduced (Figure 2B and C). After the eighth cycle, CT indicated that the image was slightly better than the previous $\mathrm{CT}$ image (Figure 2D), and the PET/CT showed increased foci of brown adipose tissue that distributed weaker radioactivity all over the body compared with previous scans. There were no significant changes on the right side of the testicular resection area compared with previous $\mathrm{PET} / \mathrm{CT}$ results. There was also no obvious tumor tissue after treatment (Figure 3). The entire treatment after eight cycles was considered as CR. In addition, there were no severe adverse reactions that appeared during the treatment, and the patient's symptoms were reduced with a Karnofsky performance score of 90 . He has been alive for 19 months since abdominal metastasis.

\section{Discussion}

Metastatic ERMS has a high mortality rate and a poor prognosis. The treatment options for non-pleomorphic (alveolar and embryonal) ERMS in the National Comprehensive Cancer Network guidelines do not describe a specific chemotherapy regimen, but emphasize that this disease type is different from other STS systemic chemotherapies, and multidisciplinary combination therapy is recommended. ${ }^{2}$

Gee et al discovered that vascular endothelial growth factor receptor and vascular endothelial growth factor (VEGF) exist in patients with ERMS. ${ }^{7}$ Moreover, VEGF has high expression and regeneration in this group. ${ }^{8,9}$ Unfortunately, VEGF binding to its receptor can promote tumor cell proliferation. Endostar is a broad-spectrum VEGF inhibitor that may inhibit tumor growth in ERMS. ${ }^{10}$ Although immediate reports are lacking, much of the research on STSs has examined this option. In a study of advanced bone and STSs, Endostar combined with chemotherapy had significant activity toward increasing PFS and improving the clinical benefit rate. ${ }^{11}$ Teng et al reported that the treatment of a pleural synovial sarcoma patient with combined chemotherapy and Endostar plus sunitinib was effective. ${ }^{12}$ A retrospective case series study with a total of 71 

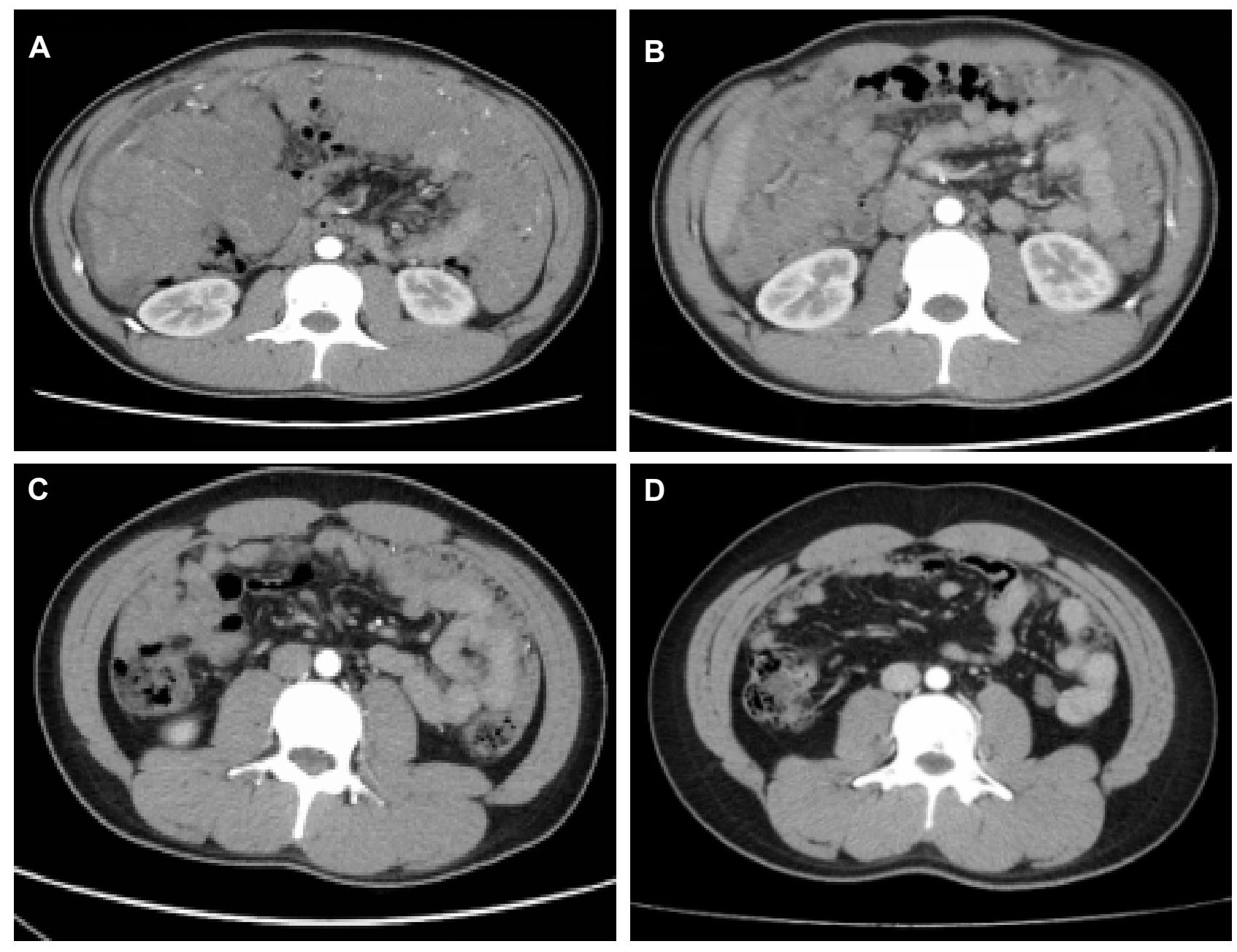

Figure 2 Whole abdomen CT images show abdominal mass prior to treatment (A), after 2 cycles of treatment (B), after 4 cycles of treatment (C), and after the whole treatment (8 cycles) (D).

patients suffering from advanced STSs suggested that Endostar combined with chemotherapy had a higher disease control rate and longer PFS than traditional chemotherapy. ${ }^{13}$

For ERMS, it is recommended that vincristine, actinomycin $\mathrm{D}$, cyclophosphamide, isocyclophosphamide, adriamycin, cisplatin, and irinotecan are applicable, but there is no first-line protocol. ${ }^{14,15}$ The VAC (vincristine, actinomycin D, and cyclophosphamide) and IVA (ifosfamide, vincristine, and actinomycin D) regimens are the most widely used for adjuvant chemotherapy, but patients develop metastatic disease after adjuvant chemotherapy and are treated with multidrug chemotherapy. ${ }^{16}$ For the treatment of relapsed disease, a clinical trial (NCT01355445) using vincristine, irinotecan, and temozolomide may provide hope for patients with refractory/relapsed rhabdomyosarcoma.

In this study, our patient, who used Endostar combined with cisplatin and isocyclophosphamide, did not follow the routine chemotherapy regimen because in the genetic test results, cis-platinum had a good curative effect and low toxicity. To our surprise, after eight cycles of treatment, he was evaluated as having CR, and PFS was 19 months rather than the average PFS of 13.3 months seen with the routine chemotherapy regimen of vincristine, d-actinomycin, and cyclophosphamide. ${ }^{17}$ In a study by Liu et al,

Table I Specific chemotherapy of the patient

\begin{tabular}{lllll}
\hline Chemotherapy regimen & Dosage & Delivery route & Time & Interval \\
\hline Endostar & $7.5 \mathrm{mg} / \mathrm{m}^{2} /$ day & Continuous IV pumping & Days I-I4 & Every $2 \mathrm{I}$ days \\
Cisplatin & $30 \mathrm{mg} / \mathrm{m}^{2} /$ day & $\mathrm{IV}$ & Days I-3 & for eight cycles \\
Ifosfamide (Mesna) & $\mathrm{I} .2 \mathrm{~g} / \mathrm{m}^{2} / \mathrm{day}$ & $\mathrm{IV}$ & Days I-5 & \\
\hline
\end{tabular}

Abbreviation: IV, intravenous. 

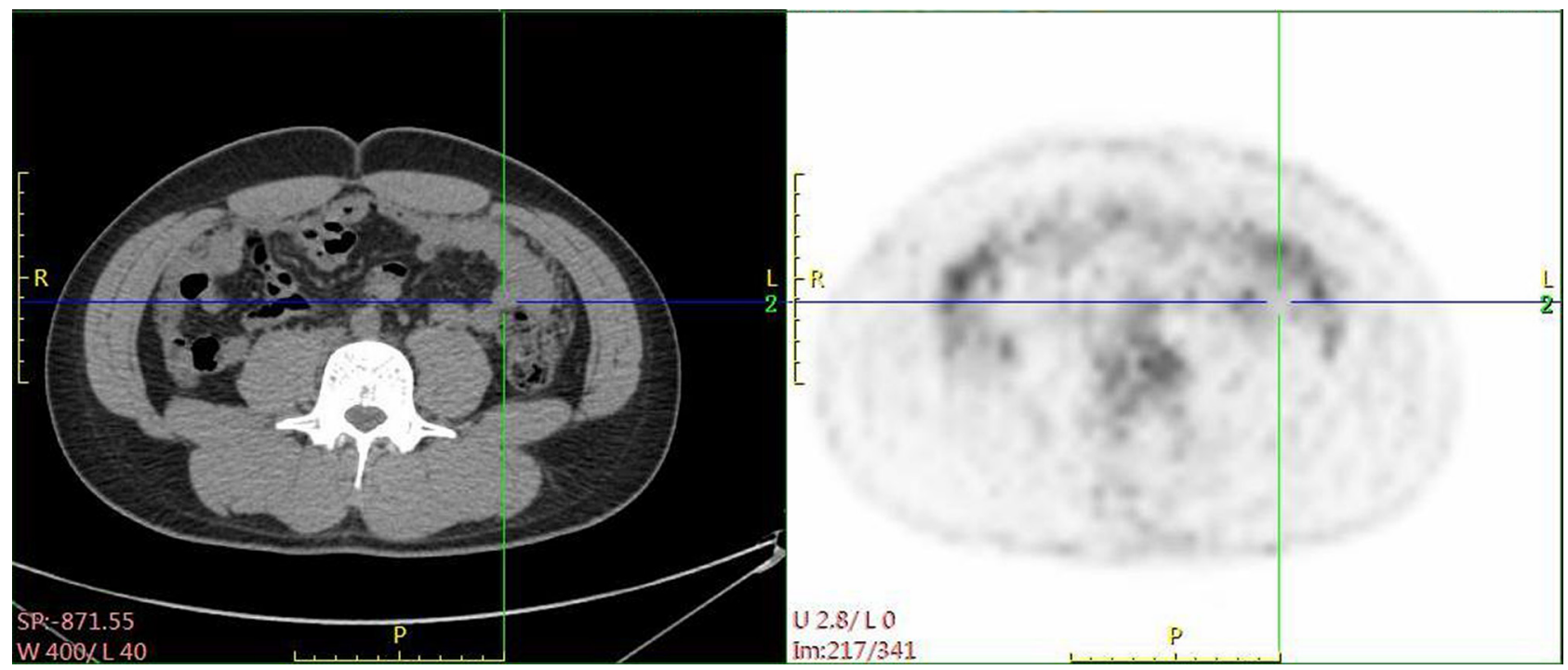

Figure 3 PET/CT images show abdomen area after the whole treatment (8 cycles).

two patients with relapse or metastasis who were treated with RIO+RPLND+chemotherapy only achieved partial response. ${ }^{18}$ To our knowledge, this is the first case to report the successful use of Endostar and chemotherapy with cisplatin and isocyclophosphamide in abdominal cavity relapsed metastasis after testicular ERMS orchiectomy. Moreover, the curative effect resulted in CR. Meanwhile, Endostar caused tolerable side effects; its adverse clinical reactions are cardiac reactions. Rarer reactions include mainly skin and accessory allergic reactions and digestive tract reactions. Endostar did not cause cardiotoxicity in our patient, and combined with chemotherapy, Endostar may play a synergistic role in the treatment of ERMS.

\section{Conclusion}

Currently, there is no definite protocol for the treatment of metastatic ERMS. Conventional chemotherapy has limited efficacy and poor prognosis. Our case report is the first report using chemotherapy and Endostar, with CR. Endostar, a new vascular targeted drug, combined with chemotherapy may play a synergistic role, but further clinical confirmation is needed.

\section{Acknowledgments}

This work was supported by the National Natural Science Foundation of China (No 81702622) and the Army Medical Science and Technology Youth Development Scheme (15QNP005).

\section{Disclosure}

The authors report no conflicts of interest in this work.

\section{References}

1. Sultan I, Qaddoumi I, Yaser S, Rodriguez-Galindo C, Ferrari A. Comparing adult and pediatric rhabdomyosarcoma in the surveillance, epidemiology and end results program, 1973 to 2005: an analysis of 2,600 patients. J Clin Oncol. 2009;27(20):3391-3397.

2. von MM, Randall RL, Benjamin RS, et al. Soft tissue sarcoma, version 2.2018. J Natl Compr Canc Netw. 2018;16(5):536-563.

3. Athbun RC, Lockhart SM, Stephens JR. Rhabdomyosarcoma in adults: new perspectives on therapy. Curr Treat Options Oncol. 2015; $16(6): 342$

4. Brennan MF, Alektiar KM, Maki RG. Cancer: Principles \& Practice of Oncology. New York: Lippincott Williams \& Wilkins; 2011.

5. Limaverde-Sousa G, Sternberg C, Ferreira CG. Antiangiogenesis beyond VEGF inhibition: a journey from antiangiogenic single-target to broad-spectrum agents. Cancer Treat Rev. 2014;40(4):548-557.

6. Folkman J. Role of angiogenesis in tumor growth and metastasis. Semin Oncol. 2002;29(6 Suppl 16):15-18.

7. Gee MF, Tsuchida R, Eichler-Jonsson C, et al. Vascular endothelial growth factor acts in an autocrine manner in rhabdomyosarcoma cell lines and can be inhibited with all-trans-retinoic acid. Oncogene. 2005; 24(54):8025-8037.

8. Schiavetti A, Mcdowell HP, Conti L, et al. Vascular endothelial growth factor serum levels in children with newly diagnosed rhabdomyosarcoma. Pediatr Blood Cancer. 2012;59(4):627-630.

9. Ganti R, Skapek SX, Zhang J, et al. Expression and genomic status of EGFR and ErbB-2 in alveolar and embryonal rhabdomyosarcoma. Mod Pathol. 2006;19(9):1213-1220.

10. Crose LES, Linardic CM. Receptor tyrosine kinases as therapeutic targets in rhabdomyosarcoma. Sarcoma. 2010, 2011;714X:1357.

11. Xing P, Zhang J, Yan Z, et al. Recombined humanized endostatin (Endostar) combined with chemotherapy for advanced bone and soft tissue sarcomas in stage IV. Oncotarget. 2017;8(22):36716-36727.

12. Teng C, Li LI, Shen W, et al. Pleural synovial sarcoma patient treated with combined chemotherapy and Endostar, plus sunitinib maintenance therapy: a case report and review of the literature. Oncol Lett. 2015; 10(2):1141-1144.

13. Zhang LP, Liao XY, Xu YM, et al. Efficacy and safety of Endostar ${ }^{\circledR}$ combined with chemotherapy in patients with advanced soft tissue sarcomas. Asian Pac J Cancer Prev. 2013;14(7):4255-4259.

14. Ruymann FB. The development of VAC chemotherapy in rhabdomyosarcoma: what does one do for an encore? Curr Oncol Rep. 2003;5(6): $505-509$. 
15. Oberlin O, Rey A, Lyden E, et al. Prognostic factors in metastatic rhabdomyosarcomas: results of a pooled analysis from United States and European cooperative groups. J Clin Oncol. 2008;26(14): 2384-2389.

16. Zhan XK, Zhang S, Cao BW, et al. Clinicopathological characteristics and treatment outcomes of Chinese patients with genitourinary embryonal rhabdomyosarcoma. World J Surg Oncol. 2015;13(1):190.
17. Kojima Y, Hashimoto K, Ando M, et al. Clinical outcomes of adult and childhood rhabdomyosarcoma treated with vincristine, d-actinomycin, and cyclophosphamide chemotherapy. J Cancer Res Clin Oncol. 2012; 138(7):1249-1257.

18. Liu ZW, Zhang XQ, Hou GL, et al. Primary adult intratesticular rhabdomyosarcoma: results of the treatment of six cases. Int J Urol. 2011;18(2):171-174.

\section{Publish your work in this journal}

OncoTargets and Therapy is an international, peer-reviewed, open access journal focusing on the pathological basis of all cancers, potential targets for therapy and treatment protocols employed to improve the management of cancer patients. The journal also focuses on the impact of management programs and new therapeutic agents and protocols on

\section{Dovepress}

patient perspectives such as quality of life, adherence and satisfaction. The manuscript management system is completely online and includes a very quick and fair peer-review system, which is all easy to use. Visit http://www.dovepress.com/testimonials.php to read real quotes from published authors.

Submit your manuscript here: http://www.dovepress.com/oncotargets-and-therapy-journal 